\title{
Convective instability induced by two-points nonlocality
}

\author{
Roberta Zambrini and Francesco Papoff \\ SUPA, Department of Physics, University of Strathclyde, 107 Rottenrow, Glasgow G4 0NG, United Kingdom
}

(Received 5 November 2005; published 23 January 2006)

\begin{abstract}
We consider diffusive nonlinear systems with nonlocal two-points coupling, generally induced by misalignment in optical feedback. We expand the stability analysis in F. Papoff and R. Zambrini [Phys. Rev. Lett. 94, 243903 (2005)] to determine convective and absolute thresholds. Nonlocality leads to different effects in comparison to well-known problems with drift, as the existence of opposite phase and group velocities for some modes and an instability region. The theoretical predictions are in agreement with numerical results in a nonlocal system with saturable nonlinearity over wide parameter regions. The knowledge of the stability diagram for any uniform state allows us to interpret the rich dynamics due to the interplay between finite size, noise, and multiple states.
\end{abstract}

DOI: 10.1103/PhysRevE.73.016611

PACS number(s): 42.65.Sf, 05.40.Ca, 89.75.Kd

\section{INTRODUCTION}

Important dynamical effects induced by nonlocality have been recently reported, both in the temporal domain and in the spatial one, in systems with long-range correlations. Often these effects are modeled by integrodifferential equations; some examples include dispersive [1] and photorefractive materials [2], Bose-Einstein condensates [3], and population dynamics [4]. A fundamental kind of nonlocality arises by coupling any point in the system with a shifted one, leading to a discrete two-points coupling. In the temporal domain, a two-points coupling would model, for instance, a laser device with delayed feedback [5]. In the spatial domain, two-points nonlocal coupling arises naturally in any optical device with light confined by cavities or mirrors, whenever the optical elements providing the feedback are not perfectly aligned [7-9]. Indeed, the field in a point $x$ in the medium is coupled with the field in a shifted point $x+\Delta x$. It is worth to stress that other often-called nonlocal terms, such as spatial derivatives (i.e., drift, diffusion, and diffraction terms) [6], lead to an infinitesimal coupling range, whereas the twopoints nonlocality considered here has a finite and arbitrarily large coupling distance $\Delta x$.

Specific effects of two-points nonlocality have been reported in several nonlinear optical systems with not aligned feedback. Examples are experiments with sodium vapors [7] as well as liquid-crystal light valves [8] and liquid crystals [9]. An observed effect of the misalignment is the traveling character of the spatial pattern formed in the transverse profile of the light beam, making these devices good candidates for convective instabilities [10-13]. These instabilities have been recognized in a wide class of systems, in plasma physics [14], fluids [15], optics [16], surface science [17], chemical reactions [18], and traffic [19]. Whenever a localized disturbance of an unstable state does drift, two possible scenarios can arise: if the perturbation grows locally the instability is absolute, while if the perturbation spreads slower than it drifts, decaying locally, the instability is convective. The existence of these instabilities has been shown in systems whose dynamics is characterized by drift or walk off, modeled by a gradient term [11-16]. Only recently these instabilities have been experimentally observed in a mis- aligned optical system [9] and theoretically predicted in a large class of diffusive systems with two-points coupling [20].

In this paper we extend the analysis of Ref. [20], discussing the main aspects of the stability analysis of nonlinear diffusive equations with nonlocal coupling in comparison to the case of drift modeled by gradient. Section II is devoted to the theoretical linear stability analysis. The complexity and peculiarities of the dispersion relation are discussed both locally and globally in order to obtain the correct stability diagram. Phase and group velocities are compared and shown to be of opposite sign for the critical mode. We also show by a counterexample that local criteria based on the second-order derivative of the dispersion are neither sufficient nor necessary to find the points relevant for the evaluation of the absolute threshold.

In Sec. III, we study, numerically, the rich dynamics of the nonlocal saturable nonlinear equation in different regimes. These results not only confirm some quantitative predictions of our general analysis (as thresholds position, most unstable modes, phase and group velocities) but allow us also to see in a huge parameter region to which extent the presented general linear stability can be useful to interpret the spatiotemporal dynamics of the specific model here considered.

\section{NONLOCAL DIFFUSIVE SYSTEM}

We consider equations of the type

$$
\left(\partial_{t}-\partial_{x}^{2}\right) \phi(x, t)=f_{1}(\phi(x, t) ; \mu)+f_{2}(\phi(x+\Delta x, t) ; \mu),
$$

where $\phi$ is a real variable, $t$ is in units of the diffusion time, $x$ and the spatial shift $\Delta x$ are in unit of the diffusion length, and $\mu$ is a control parameter independent on $x . f_{1}, f_{2}$ are real functions that can be derived with respect to $\phi$. The uniform states $\phi_{m}$ of Eq. (1) are the solutions of $f_{1}+f_{2}=0$, and their domains of existence depend on $\mu$ but not on $\Delta x$ in the limit of infinitely extended systems. The dispersion relation for perturbations $\exp \left(\omega t+i k_{I} x\right)$ of a uniform state of Eq. (1) is 


$$
D\left(\omega, k_{I}\right)=\omega+k_{I}^{2}-\partial_{\phi} f_{1}\left(\phi_{m} ; \mu\right)-\partial_{\phi} f_{2}\left(\phi_{m} ; \mu\right) e^{i k_{I} \Delta x}=0 .
$$

As a consequence of the term $e^{i k_{1} \Delta x}$, which is present in all systems with shift, there are bands of $k_{I}$ for which the real part of the dispersion relation $\omega_{R}$ can be positive. For $\partial_{\phi} f_{1}\left(\phi_{m} ; \mu\right)<0$, as in the experiments in Ref. [8], these bands are within the regions where $\partial_{\phi} f_{2}\left(\phi_{m} ; \mu\right) \cos k_{I} \Delta x>0$. As a result, the homogeneous solution $\phi_{m}$ is unstable and plane wave perturbations are amplified. As the imaginary part of $\omega$ (phase velocity) is, in general, non-null, these waves move across the system. For each unstable band, the wave number of the most unstable perturbation is implicitly given by

$$
k_{I C}=-\frac{1}{2} \partial_{\phi} f_{2}\left(\phi_{m} ; \mu\right) \Delta x \sin k_{I C} \Delta x .
$$

From this, we find that the phase and group velocities, $v_{\mathrm{ph}}$ and $v_{G}$, of the most unstable mode are

$$
\begin{gathered}
v_{\mathrm{ph}}=-\frac{\omega_{I}\left(k_{I C}\right)}{k_{I C}}=\frac{2}{\Delta x}, \\
v_{G}=-\left.\partial_{k_{I}} \omega_{I}\right|_{k_{I C}}=-\partial_{\phi} f_{2}\left(\phi_{m} ; \mu\right) \Delta x \cos k_{I C} \Delta x .
\end{gathered}
$$

Equations (4) and (5) show two peculiar effects of the nonlocality: for each band the phase velocity of the most unstable perturbation depends only on the shift $\Delta x$ (in units of the diffusion length) and not on the nonlinear functions $f_{1}, f_{2}$; for spatially localized perturbations the sign of the group velocity and of the phase velocity of the most unstable wave number are always opposite. Given the phase velocity for a generic mode $v_{\mathrm{ph}}=\partial_{\phi} f_{2}\left(\phi_{m} ; \mu\right) \sin \left(k_{I} \Delta x\right) / k_{I}$, it also follows that, if $\partial_{\phi} f_{2}\left(\phi_{m} ; \mu\right)<0$, a perturbation with $k_{I}=\pi / \Delta x$ becomes unstable with null phase velocity when $\partial_{\phi} f_{1}-\partial_{\phi} f_{2}$ $=(\pi / \Delta x)^{2}$. Beyond this point, the instability band contains perturbations with positive, negative, and null phase velocity.

When the group velocity is nonvanishing, one has to consider whether localized perturbations produce absolute or convective instability. We have shown that nonlocality can be at the origin of convective instabilities in a wide class of diffusive systems, similarly to what was observed with drift [20], and it is worth here to analyze similarities and differences between these two classes of systems. In the limit of small shift, the field in the point $x+\Delta x$ can be approximated by $\phi(x+\Delta x) \simeq \phi(x)+\Delta x \partial_{x} \phi$ and this might suggest that both terms lead to similar effects. However, a fundamental difference appears when we compare the dispersion in (2) to the dispersion relation for a diffusive system with shift,

$$
\omega=a-k_{I}^{2}+i v k_{I},
$$

with $a$ constant depending on control parameters and homogeneous solutions. The walk-off term leads to a purely imaginary contribution to the dispersion relation being at the origin of Hopf instabilities. On the other hand, the nonlocal coupling contributes both to the real and imaginary parts of $\omega$, influencing also the instability thresholds. Therefore, nonlocality allows for the existence of new regimes of convective as well as absolute instability, not accessed when drift effects instead of nonlocal couplings are considered in the same system.

Beside the dispersion relations, a common feature of both drift and shift is that the reflection symmetry $x \rightarrow-x$ is broken, but there is also a fundamental difference. Given one dynamical equation, a drift term disappears in the proper moving reference frame, so that stationary solutions of the equation without drift lead immediately to the traveling solutions of the system with drift. On the other hand, a nonlocal coupling cannot be eliminated in any reference frame and this leads to surprising effects, such as the possibility to observe phase and group velocities with opposite sign. Traveling patterns that cannot be reduced to stationary states in any reference frame have also been shown to be able to exhibit nonlinear waves modulations [23].

Important differences between problems with nonlocality and with drift appear already in the linear stability analysis. In the following, we show that the stability diagrams reported in Ref. [20] can be rigorously derived by using the saddle-point technique. To find the nature of the instability, we determine whether, in the laboratory frame, the perturbation caused by an impulse $\delta(x) \delta(t)$ fills the entire system or disappears for large times. Mathematically, this means to find whether the Green's function of the linearized equation,

$$
G(x, t)=\frac{1}{(2 \pi)^{2}} \int_{\gamma-i \infty}^{\gamma+i \infty} \int_{-\infty}^{+\infty} \frac{e^{i k_{I} x+\omega t}}{D\left(\omega, k_{I}\right)} d k_{I} d \omega,
$$

with $\gamma$ a real constant, diverges or vanishes for $t \rightarrow+\infty$ $[12,24]$. To evaluate the integral, we integrate first in $\omega$, using a $\gamma$ such that all the branches $\omega\left(k_{I}\right)$ with $D\left(\omega\left(k_{I}\right), k_{I}\right)$ $=0$ lie on the left the $\omega=\gamma$ contour. By appropriately closing the contour with infinite semicircles and using residue evaluation [24], we find that $G(x, t)$ vanishes for $t<0$, while for $t>0$ we have

$$
G(x, t)=\frac{-i}{(2 \pi)} \int_{-\infty}^{+\infty} \frac{e^{i k_{I} x+\omega\left(k_{I}\right) t}}{\partial_{\omega} D\left(\omega\left(k_{I}\right), k_{I}\right)} d k_{I} .
$$

In our case, $\partial_{\omega} D=1$ as $D\left(\omega, k_{I}\right)$ is linear in $\omega$. In order to estimate Eq. (8), it is convenient to extend analytically $\omega\left(k_{I}\right)$ in the complex plane $k=k_{R}+i k_{I}$ and apply the saddle-point method. An even number (at least four) of paths with $\omega_{I}$ constant start from each saddle point with $d \omega / d k=0$, as shown in Fig. 1. On half of these equiphase paths, the steepest descents, $\omega_{R}$ decreases fastest; on the remaining half, the steepest ascents, $w_{R}$ increases fastest. If one can form a closed integration contour with the imaginary axis and steepest descents, then the asymptotic value of the integral is given by the values of $\omega$ at the saddle points on the integration contour [21].

This method has been extensively used to find the threshold between convective and absolute instabilities in systems with drift, where the dispersion has, in general, few saddle points. In the case of drift (6), there is indeed only one saddle point. However, for nonlocal systems, the exponential term in the dispersion (2) originates always a countable infinity of saddle points. Moreover, the saddles and their steepest descents move and can suddenly collide and disappear as the 


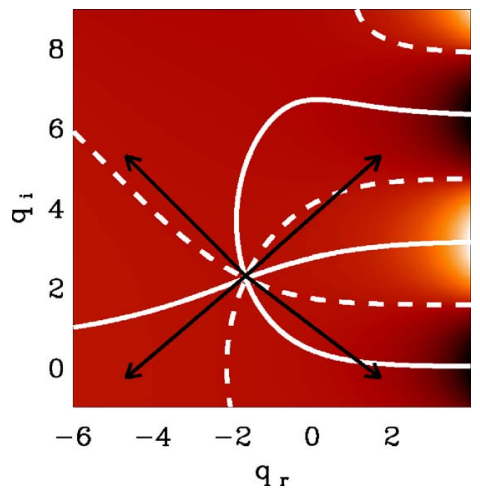

FIG. 1. (Color online) Real part of the dispersion relation for $\chi^{\prime}=-30$ in the region of the saddle point $s_{0}$. The most intense regions have light colors, and both equiphase (continuous) and equiamplitude (dashed) lines are represented. The arrows delimit a wedge of $(-\pi / 4, \pi / 4)$ around the parallel to the imaginary axis. The steepest descent path at the saddle point is contained in this wedge because it satisfies the condition $\operatorname{Re}\left\{d^{2} \omega / d k^{2}\right\}>0$.

control parameters change. It is therefore essential to determine if one can limit the analysis to a finite number of saddle points and, if this is possible, to find which of these points have equiphase paths that correctly close the integration contour as the control parameters change. In the following, we solve both of these problems by studying how the local and global geometrical organization of the saddles and of the equiphase paths changes with the control parameters.

We note that one could have chosen to integrate Eq. (7) first in $k$. In this case, one does not apply the saddle-point technique, but only the residue theorem, and the stability of the system is determined by the local and global properties of branch points where two or more different branches $k_{n}(\omega)$, with $D\left(\omega, k_{n}(\omega)\right)=0$, intersect, as explained in Ref. [25]. Because also the number of branch points is infinite (they turn out to coincide with the saddle points), the main problem is again to find a limit to the number of points that need to be analyzed, with the further difficulty that in our case there is not an explicit equation for the branches $k_{n}(\omega)$. As a final remark, we have found in the recent literature, the condition $\operatorname{Re}\left\{d^{2} \omega / d k^{2}\right\}>0$ used to identify the saddle points relevant to the stability. We show in the following that this procedure, in general, does not correctly select the saddles whose equiphase paths close the integration contour; these points are instead identified by the global analysis described here.

From here onward, we consider the analytic extension of the dispersion relation

$$
w=\xi \Delta x^{2}+q^{2}+\chi \Delta x^{2} e^{q},
$$

with $w=\omega \Delta x^{2}, q=k \Delta x$, and parameters $\xi=\partial_{\phi} f_{1}\left(\phi_{m} ; \mu\right), \chi$ $=\partial_{\phi} f_{2}\left(\phi_{m} ; \mu\right)$. The symmetry $w(q)=w^{*}\left(q^{*}\right)$ allows us to consider only the semi-plane $q_{I} \geqslant 0$. Note that the dispersion relation and the stability depend only on the effective parameters $\xi^{\prime} \equiv \xi \Delta x^{2}$ and $\chi^{\prime} \equiv \chi \Delta x^{2}$.

In order to close the integration contour entirely with steepest descents, we need a steepest descent path that ends on or is asymptotic to the real axis $\left(q_{R}\right)$ and another asymptotic to the imaginary axis $\left(q_{I}\right)$. These steepest de- scents must either come from the same saddle or be connected by other steepest descents.

\section{A. Local analysis: Saddle points' position, motion, and order}

First of all, we need to find how many saddles, i.e., points $q=s$, that solve the equation

$$
\left.\frac{d w}{d q}\right|_{q=s}=2 s+\chi^{\prime} e^{s}=0,
$$

exist for each value of the control parameters. As previously noted, Eq. (10) is invariant under $s_{I} \rightarrow-s_{I}$; thus, for each saddle $\left(s_{R}\left(\chi^{\prime}\right), s_{I}\left(\chi^{\prime}\right)\right)$ there is also a saddle $\left(s_{R}\left(\chi^{\prime}\right)\right.$, $\left.-s_{I}\left(\chi^{\prime}\right)\right)$. Moreover, $s_{I}=0$, with appropriate $s_{R}$ and $\chi^{\prime}$, is a solution of Eq. (10) and, for all values of $\chi^{\prime}$, it must be $s_{I}$ $\neq(n+1) \pi$, with $n \in N$.

In the case $s_{I}=0$, we find that Eq. (10) has no solution for $\chi^{\prime}<-2 / e$ [Fig. 2(a)], two degenerate solutions for $\chi^{\prime}$ $=-2 / e[$ Fig. 2(b)], two nondegenerate solutions for $-2 / e$ $\leqslant \chi^{\prime} \leqslant 0$ [Fig. 2(c)] and one solution for $\chi^{\prime}>0$ [Fig. 2(d)]. For saddle points with $s_{I} \neq n \pi$, the real and imaginary parts of Eq. (10) can be recast as

$$
\begin{gathered}
s_{R}=s_{I} \cot s_{I}, \\
-\frac{2}{\chi^{\prime}} s_{I}=\sin s_{I} e^{s_{I} \cot s_{I} .}
\end{gathered}
$$

We can only analyze $s_{I}$ because $s_{R}$ is a function of $s_{I}$. We first consider the interval $s_{I} \in(0, \pi)$ : for $\chi^{\prime}<-2 / e$, Eq. (12) admits one solution, while there are no solutions for $\chi^{\prime}$ $>-2 / e$. We now introduce the notation $s_{0}\left(\chi^{\prime}\right)$ to indicate the left-most saddle on the real axis for $\chi^{\prime}>-2 / e$ and the saddle with $s_{I} \in[0, \pi)$ for $\chi^{\prime} \leqslant-2 / e$. For all $n>0$, there is also one solution $s_{n}$ in the interval $(2 n \pi,(2 n+1) \pi)$ for $\chi^{\prime}<0$ and in the interval $((2 n+1) \pi,(2 n+2) \pi)$ for $\chi^{\prime}>0$.

We consider now how the saddle points move as $\chi^{\prime}$ varies. When $\chi^{\prime}$ decreases from $+\infty$ to $0^{+}, s_{n I}$ decrease monotonically from $2(n+1) \pi$ to $(2 n+1) \pi$, and $s_{n R}$ increases monotonically from $-\infty$ to $+\infty$. At the same time, the saddle point on the real axis moves from $-\infty$ to $0^{-}$. For $\chi^{\prime}=0^{-}$, another saddle point appears on the on the real axis at $+\infty$; these two saddle points move toward one another until they collide in $s_{R}=1$ for $\chi^{\prime}=-2 / e$, as shown in Fig. 2(b). Two new points are then generated in the intervals $0<s_{I}<\pi$ and $-\pi<s_{I}<0$ [Fig. 2(a)] and move from $s_{R}=1$ to $s_{R}=-\infty$. In each interval $2 n \pi<s_{I}<(2 n+1) \pi$, with $n \neq 0, s_{n I}$ increases monotonically from the lower bound to the upper bound of the interval as $\chi^{\prime}$ decreases from 0 to $-\infty$. At the same time, $s_{n R}$ decreases monotonically from $+\infty$ to $-\infty$. For ease of notation, in the following we will drop the index from the saddle points whenever it is not necessary. We now determine the number of the equiphase path emerging from a saddle point by using the fact that each of these paths has a tangent at the saddle point along which, in a small neighborhood of the saddle, the phase remains constant.

From the Taylor expansion of $w$ near a saddle, 

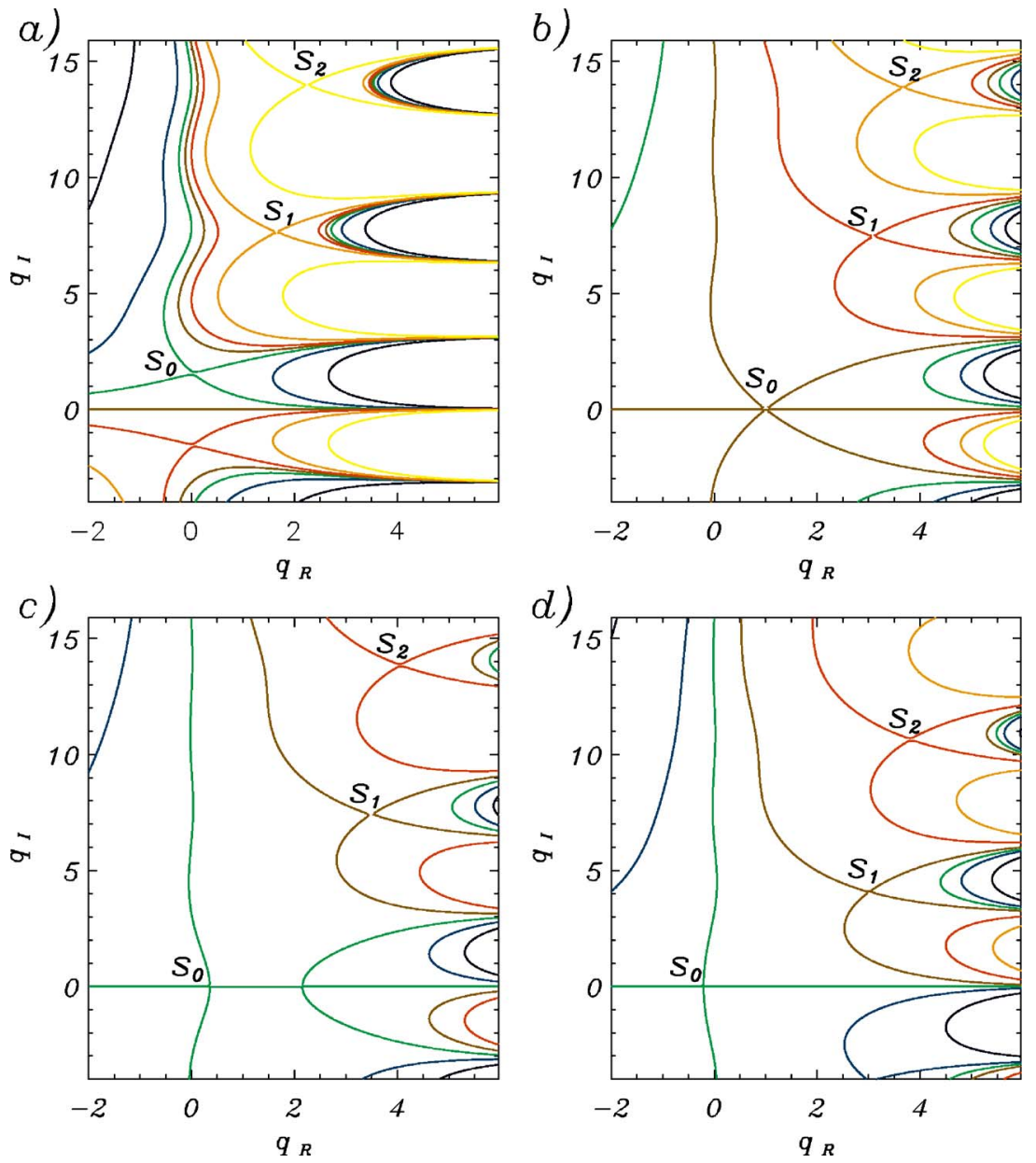

FIG. 2. (Color online) Equiphase lines $\left[2 q_{I} q_{R}+\chi^{\prime} \sin \left(q_{I}\right)\right.$ $\times \exp q_{R}=$ const $]$ for $\chi^{\prime}=-3$ (a), $-2 / e$ (b), -0.5 (c) and +0.5 (d). The labels $s_{i}$ identify the saddle points as discussed in the text.

$$
w[s+\rho \exp (i \theta)] \sim w(s)+\left.\frac{1}{m !} \frac{d^{m} w}{d q^{m}}\right|_{s} \rho^{m} e^{i m \theta},
$$

where $m$ is the order of the lowest nonvanishing derivative, one can see that there are $m$ directions along which the phase remains constant for $\rho \ll 1$. For the class of equations considered here, $\left.\quad\left(d w / d q_{I}\right)\right|_{s}=\left.\left(d^{2} w / d q_{I}^{2}\right)\right|_{s}=0$ only when $s=(1,0)$ and $\chi^{\prime}=-2 / e$. This is where the two points on the real axis merge into a single saddle with $m=3$, which has six equiphase lines: three steepest descent and three steepest ascent, as shown in Fig. 2(b). Apart from this point, all the others have $m=2$ and two steepest descent lines with tangents at $\pi / 2$ to one another and at $\pm \pi / 4$ to the tangents to the two steepest ascent lines.

\section{B. Global analysis: Equiphase and equiamplitude paths at infinity}

The equation for equiphase and equiamplitude paths emerging from the saddle $s$ are

$$
\begin{gathered}
2 q_{I} q_{R}+\chi^{\prime} e^{q_{R}} \sin q_{I}=w_{I}\left(s_{I}\right), \\
\xi^{\prime}+q_{R}^{2}-q_{I}^{2}+\chi^{\prime} e^{q_{R}} \cos q_{I}=w_{R}\left(s_{I}\right),
\end{gathered}
$$

respectively. Because the dispersion relation is analytic for all finite values of $q$, its equiphase paths can intersect each other only at saddle points if they have the same phase, or merge at $\infty$ even if they have different phases [22]. We have already found the saddle points, we now need to find the limits of the equiphase lines at $\infty$. Before doing that, note the following properties of the equiphase lines: (i) On any circle around one saddle point, including one with infinite radius, an interception of a steepest ascent with the circle must be between interceptions of two steepest descents and vice versa; (ii) only one of the equiphase paths emerging from any saddle can cross the lines $q_{I}=n \pi$, as can be seen by the fact that, for $q_{I}=n \pi, q_{R}=w_{I} / 2 n \pi$ is the only value that satisfies Eq. (14); (iii) for $w_{I}=0, q_{I}=0$ is an equiphase line; (iv) for $w_{I} \neq 0$, it must be $q_{I} \neq 0$, which means that the equiphase lines are confined in the semi-planes $q_{I}>0$ and $q_{I}<0$; and (v) for $w_{I} \neq 0, q_{R}=0$ only if $\left|w_{I} / \chi\right|<1$ [in that case, $q_{I}$ $=\arcsin \left(w_{I} / \chi^{\prime}\right)+2 n \pi$ or $\left.q_{I}=\arcsin \left(-w_{I} / \chi^{\prime}\right)+(2 n+1) \pi\right]$.

Equations (14) and (15) implicitly define $q_{R}$ as a function of $q_{I}$ and vice versa. We look then for asymptotic expressions of $q_{I}\left(q_{R}\right)$ for $q_{R} \rightarrow \pm \infty$ and $q_{R}\left(q_{I}\right)$ for $q_{I} \rightarrow+\infty$ able to fulfill asymptotically these equations. We show in the Appendix that the asymptotes of the equiphase paths are $q$ $=(+\infty, n \pi),(-\infty, 0),(0,+\infty)$. The asymptotes of the steepest descent paths are those where $w_{R} \rightarrow-\infty$, i.e., $(0,+\infty)$ for all $\chi^{\prime}$ and $(+\infty, 2 n \pi)$ for $\chi^{\prime}<0$ or $(+\infty,(2 n+1) \pi)$ for $\chi^{\prime}>0$. The remaining cases are asymptotes of the steepest ascent paths $\left(w_{R} \rightarrow+\infty\right)$. Similarly, we show that the asymptotes for the equiamplitudes paths are $(+\infty,(n+1 / 2) \pi)$ and $(-\infty, \pm \infty)$. 


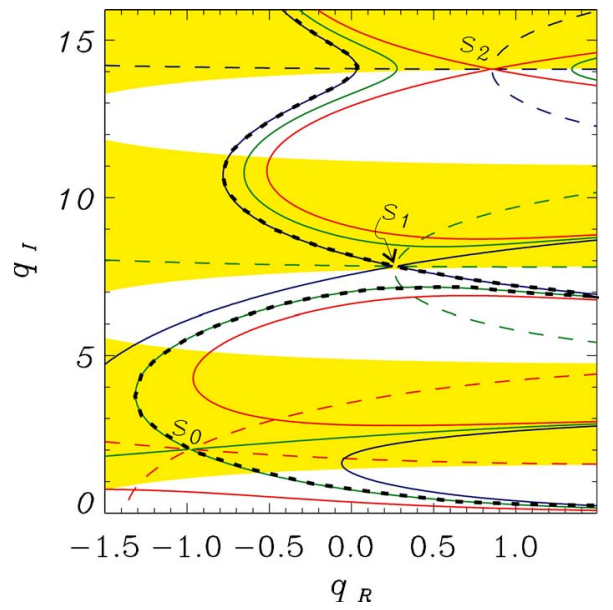

FIG. 3. (Color online) Equiphase (continuous) and equiamplitude (dashes) lines for $\chi^{\prime}=-12$ and $\xi^{\prime}=-1$ [see Eq. (9)]. The closing path is marked with symbols. Even if the (three) shown saddle points $s_{i}(i=0,1,2)$ all satisfy the condition $\operatorname{Re}\left\{d^{2} \omega / d k^{2}\right\}>0$ [colored (shaded) region], $s_{2}$ is actually not in the integration path.

This information allows us to determine the geometrical organization of the steepest descents. From Eq. (A1), we see that the saddle with the smallest phase $w_{I}\left[s_{1}\right.$ in Fig. 3 and $s_{0}$ in Figs. 2(a)-2(d)] has always the upper steepest descent to the left of all the other steepest descents and is asymptotic to the imaginary axis. Indeed, this saddle is always necessary to close the integration contour. If $s_{0}$ happens to be the saddle with the smallest phase, then the steepest descents of $s_{0}$ close the integration contour because $s_{0}$ either lies on the real axis [Figs. 2(b)-2(d)] or has another steepest descent asymptotic to the real axis [Fig. 2(a)]. If instead there is another saddle $s_{n_{1}}$ with $w_{I}\left(s_{n_{1}}\right) \leqslant w_{I}\left(s_{0}\right)$ (for instance the saddle $s_{1}$ in Fig. 3), than we find from Eq. (A3) that the upper steepest descent from $s_{0}$ remains below $s_{n_{1}}$ and is connected to the steepest descent from $s_{n_{1}}$ at infinity (in Fig. 3, these two steepest descents are asymptotically connected at $q_{I}=\pi$ ). In this case, we use a steepest descent from $s_{n_{1}}$ to reach values of $q_{I}$ above $s_{n_{1}}$ itself and a steepest descent from $s_{0}$ to reach the real axis. If $s_{n_{1}}$ is the saddle with the smallest phase, then the steepest descents of $s_{0}$ and $s_{n_{1}}$ close the integration contour (symbol lines in Fig. 3), otherwise we will have to include also the first saddle, $s_{n_{2}}$, with $n_{2}>n_{1}$ and $w_{I}\left(s_{n_{2}}\right) \leqslant w_{I}\left(s_{n_{1}}\right)$. In order to find the saddles to be used to close the integration contour, we repeat this process, including all the saddles whose phase is the minimum of the phases of the saddles below them. Because $w_{I}\left(s_{0}\right) \leqslant 0$, only saddles with null or negative phase can be used to close the integration contour, although not all of these saddles can be used. Furthermore, one can show that if $w_{I}\left(s_{\left.n_{X^{\prime}}\right)}\right)>0$, we have $w_{I}\left(s_{n}\right)>0$ for all $n>n_{\chi^{\prime}}$. Therefore, for each finite $\chi^{\prime}$, this procedure gives the finite set of saddles

$$
P_{\chi^{\prime}}=\left(s_{n} \mid 0 \leqslant n \leqslant n_{\chi^{\prime}}, w_{I}\left(s_{n}\right)=\min _{0 \leqslant j \leqslant n}\left\{w_{I}\left(s_{j}\right)\right\}\right),
$$

whose steepest descent paths close the integration contour.
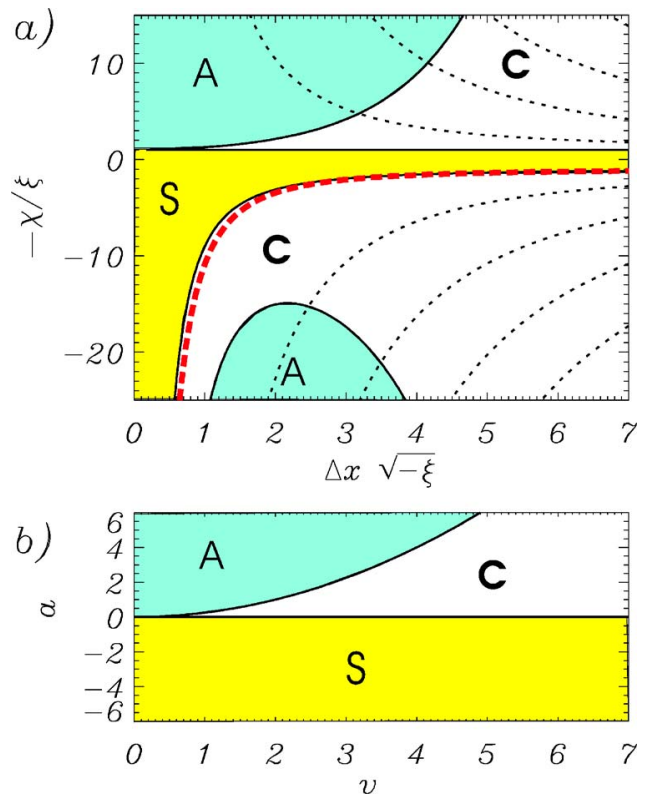

FIG. 4. (Color online) Instabilities diagram: Stable (S), convectively unstable (C) and absolutely unstable (A) regions in the cases of two-points coupling (a) and drift (b). The dispersion relations Eq. (2) with $\xi<0$ and Eq. (6) are considered, respectively. The dashed (red) line in (a) is the instability threshold for modes with vanishing phase velocity, while the dotted lines indicate where different wavenumbers bands become unstable.

From Eq. (2), we identify the region $w_{R}\left(0, q_{I}\right)<0$ in which the homogeneous solutions are stable. This is the region $\mathrm{S}$ in Fig. 4 and its contour is the convective threshold. If $w_{R}\left(0, q_{I}\right)>0$ for some $q_{I}$ and if at least one saddle $s_{n} \in P_{\chi^{\prime}}$ has $w_{R}\left(s_{n}\right)>0$, the instability is absolute. However, we need to find only a small subset of saddles in $P_{\chi^{\prime}}$ to determine the stability because $w_{R}\left(s_{n}\right)>0$ only if there is instability with $w_{R}\left(0, q_{I}\right)>0$ for $q_{I}$ in the nth interval. This follows from the fact that, for $s_{n} \in P_{\chi^{\prime}}, w_{R}\left(s_{n}\right)>0$ only if $s_{n R}<0$ and that, for $s_{n R}<0$, there is a path with $w_{R}$ constant that connects the saddle with a point on the imaginary axis within the $n$th interval. Therefore, for each $\mu$, we can determine the nature of the instability by finding the values of $w$ at the saddles in $P_{\chi^{\prime}}$ that are in bands with instability threshold $\chi_{n}^{\prime c}$ below the threshold $\chi_{0}^{\prime a}$ where $w_{R}\left(s_{0}\right)=0$. The absolute threshold $\chi^{\prime a}$ is then the value of $\chi^{\prime}$ where

$$
\max \left\{w_{R}\left(s_{n}\right) \mid s_{n} \in P_{\chi^{\prime}}, \chi^{\prime c}{ }_{n} \leqslant \chi^{\prime a}{ }_{0}^{a}\right\}=0 .
$$

The importance of Eqs. (16) and (17) is twofold. On the one hand, they guarantee that we can apply the method of steepest descents by properly closing the integration contour; on the other hand, they allow us to find the absolute threshold simply by inspection of $w$ at a finite number of saddles. This is remarkable in view of the infinite number of saddles produced by the shift. Moreover, $P_{\chi^{\prime}}$ is the same for all systems with the same $\chi^{\prime}$ in the class considered because the global geometrical organisation of the saddles and of their steepest descents does not depend on $\xi$. 
We now consider the condition $\operatorname{Re}\left\{d^{2} \omega / d k^{2}\right\}>0$ : by using Eq. (10), we find that this condition is fulfilled if and only if $s_{R}<1$ (an example is given in Fig. 1). This condition is not satisfied by $s_{0}$ for $\chi^{\prime}=-2 / e$ : for this value $s_{0}$ is degenerate, but, nevertheless, its steepest descent paths close the integration contour. Furthermore, when $s_{I}>0$, we can see from the identity $\omega_{I}(s)=2 s_{I}\left(s_{R}-1\right)$ that $\operatorname{Re}\left\{d^{2} \omega / d k^{2}\right\}>0$ is equivalent to $\omega_{I}(s)<0$. Comparing this to the previous analysis, we find many saddle points that fulfill this condition, but whose steepest descents are not part of the integration contour. An example is the saddle $s_{2}$ in Fig. 3. Therefore, the condition of the positive concavity of the dispersion relation does not distinguish the relevant saddle points to obtain the absolute threshold. Only the global analysis of the steepest descent paths correctly identifies the points that have to be used in the evaluation of the Green's function with the saddle point technique.

Applying this technique, we obtain the instabilities diagram in Fig. 4 valid for any $\xi \leqslant 0$ (including the typical case of a linear damping term $\xi=-1$ ). For $\chi<0$, the instability bands have $q_{I} \neq 0$ and the lowest convective threshold is very far from the absolute threshold. For $\chi>0$, the instability with respect to perturbations with $q_{I}=0$ is absolute for $\xi=0$ and convective for $\xi \neq 0$, with the convective instability windows increasing as $\xi$ decreases. Comparing the instabilities diagram of diffusive systems with finite shift to those with walk-off [13] (Fig. 4), we note that the whole modulation instability region for negative values of $\chi$ is a specific effect of a finite shift, as was already recognized by [8]. What our analysis reveals by direct calculation of the absolute threshold is that, in this parameter region, the system is mainly convectively unstable and shows noise-sustained modulated patterns. Only for very negative values of $\chi /|\xi|$ is the absolute threshold crossed.

It is important to extend this analysis to systems moving with velocity $v$ with respect to the laboratory frame in order to find the velocities of the instability fronts in the linear regime. The velocities of the leading and trailing fronts are the velocities of the reference frames in which the Green's function does not grow or decay [24]. Using a the rescaled velocity $v^{\prime}=v \Delta x$, the dispersion relation in the moving frame is

$$
w=\xi^{\prime}-v^{\prime} q+q^{2}+\chi^{\prime} e^{q} .
$$

We hasten to say that the analysis of Eq. (18) along the lines of what was done previously is necessary, but does not require new calculations. By defining $\widetilde{q}=q-v^{\prime} / 2, \widetilde{\xi}=\xi^{\prime}$ $-\left(v^{\prime} / 2\right)^{2}$, and $\tilde{\chi}=\chi^{\prime} e\left(v^{\prime} / 2\right)$, we recast Eq. (18) in the same form as the dispersion relation in the laboratory frame. Therefore, for each couple of values $\left(\xi^{\prime}, \chi^{\prime}\right)$, we find the velocities of the instability fronts by determining the values of $(\tilde{\xi}, \widetilde{\chi})$ corresponding to the absolute threshold in Eq. (17). This concludes the analysis of the linear stability in this class of systems.

\section{NONLOCAL SATURABLE NONLINEAR EQUATION} tion

We consider the nonlocal saturable nonlinear (NSN) equa-

$$
\left(\partial_{t}-\partial_{x}^{2}\right) \phi(x, t)=-\phi(x, t)+\mu \frac{\phi(x+\Delta x, t)}{1+\phi(x+\Delta x, t)^{2}} .
$$

This is a particular case of Eq. (1) obtained when $f_{1}=-\phi$ and $f_{2}=\mu \phi / 1+\phi^{2}$. Saturable nonlinearities, are well known to model the interaction between light and matter [26] as well as biological systems [27]. Our choice of this model is due the advantage of having a nonlinear term bounded for any value of $\phi$, being $\left|f_{2}\right| \leqslant \mu / 2$. Therefore, the dynamical model (1) is found to be always stable, even in the presence of nonlocality. As a matter of fact, some care is, in general, necessary in the choice of the nonlocal dynamical model in order to avoid divergent trajectories. If, for instance, the nonlinear term of a stable Ginsburg-Landau equation is acting nonlocally, on a shifted point of the field, the solutions are found to diverge for some parameters [20]. Otherwise, an example of a stable nonlocal model, which actually has a bounded $f_{2}$, is the liquid-crystal light valve [8].

Because of the saturable nonlinearity, Eq. (19) has three uniform states

$$
\phi_{0}=0, \quad \phi_{ \pm}= \pm \sqrt{\mu-1},
$$

with the vanishing solution always well defined and $\phi_{ \pm}$existing only for $\mu \geqslant 1$. From the Jacobian evaluated in these states, we obtain the relevant parameters

$$
\begin{gathered}
\chi_{0}=\mu, \\
\chi_{ \pm}=\frac{2-\mu}{\mu} .
\end{gathered}
$$

Given $\chi_{0, \pm}(\mu)$ and $\xi=-1$, the instability diagram in Fig. 4 provides the thresholds of the NSN in terms of the specific parameters $\mu$ and $\Delta x$. We note that the function $\chi_{ \pm}$is actually bounded to take the values in the interval $(-1,1]$. Therefore, it follows from Fig. 4 that the homogeneous states $\phi_{ \pm}$are always stable in their regime of existence $(\mu \geqslant 1)$. On the other hand, all the instability in Fig. 4 are predicted for the state $\phi_{0}$ (existing for any $\mu$ ).

In the following, we study, numerically, the dynamics and the spatial structures emerging in this system as effect of the nonlocal spatial coupling. Noise-sustained structures are observed by considering a source of noise in the NSN equation (19). As a general remark, it is important to remember that there is no convectively unstable regime if the boundary conditions for any Eq. (1) are periodic [13]. Convective instability can be observed with Dirichlet boundary conditions for the field $[\phi(-L)=\phi(L)=0$, with $2 L$ size of the system $]$ as well as by fixing the reference frame through a spatially dependent control parameter $\mu(x)$ not vanishing only within a finite region. In every simulation result presented in the following, we specify which of the two approaches is used.

\section{A. Case $\mu<1$}

For control parameter $\mu<1$, Eq. (19) admits only one homogeneous steady state $\phi_{0}$. In this regime, the parameter $\chi_{0}$ [Eq. (21)] takes the values $(-\infty, 1]$. This allows us to explore the large region of convective instability first pre- 


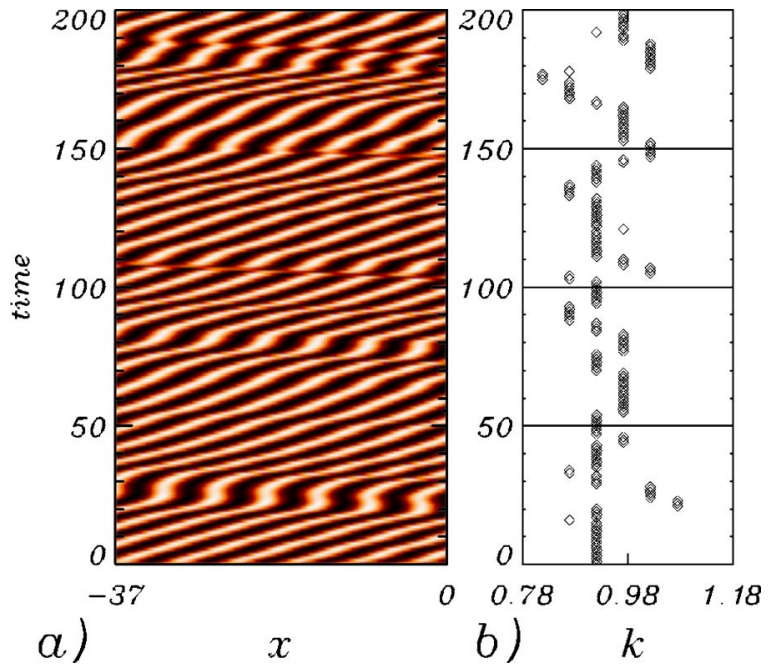

FIG. 5. (Color online) Noise-sustained pattern obtained from simulation of Eq. (19) with a white Gaussian noise. (a) Section $-37<x<0$ of the spatiotemporal diagram of the near field $\phi(x, t)$. (b) Most intense wave vector $k$ at each time. The control parameter $\mu$ has a super-Gaussian profile varying between 0 and -4 and exponent 10; noise amplitude 0.01, shift $\Delta x=2.88$. Simulation with temporal grid 0.001 , spatial grid 0.12 .

dicted in Ref. [20] where an example of noise-sustained stripe pattern was also shown. Here we detail some characteristics of these patterns, also as a function of the control parameter.

First of all, we have confirmed the position of the convective threshold for the homogeneous state: in particular, for shift $\Delta x=2.88$ the predicted theoretical threshold occurs for $\mu=-2.06$. Numerical simulations in a large system, allowing a localized perturbation of the vanishing state to grow or vanish before to reach the boundaries, show that an initial Gaussian perturbation evolves in a wave packet; for $\mu$ $=-2.1$, after a transient dynamics, the wave-packet maximum increases exponentially till saturate, while it vanishes everywhere for $\mu=-2$. The traveling wave packet developed after perturbing $\phi_{0}$ is modulated, and for $\mu=-2.1$, the most intense mode in the far field is $k \sim 0.98$. This value is in agreement with the theoretical maximally amplified wave vector (3).

A source of noise is able to sustain a pattern in this regime, as shown in Fig. 5. This noise-sustained structure shows not only strong changes of wavelength [Fig. 5(b)] but also important variations of the phase velocity that can be recognized by the change of steepness of the stripes in the spatiotemporal diagram [Fig. 5(a)]. Indeed, several simulations show that waves can travel through the systems not only at different velocities, but also in different directions, being in some cases almost stationary. Comparing to the theoretical predictions of our stability analysis, we note that all the observed wave vectors in Fig. 5(b) are linearly amplified with positive phase velocities $v_{\mathrm{ph}}=-\mu \sin \left(k_{I} \Delta x\right) / k_{I}$ (waves propagating in the right direction), as observed by numerical simulation of the full nonlinear model. In particular, the linear analysis predicts a phase velocity approaching a vanishing value for larger wave vectors, changing sign for $k \sim 1.1$.

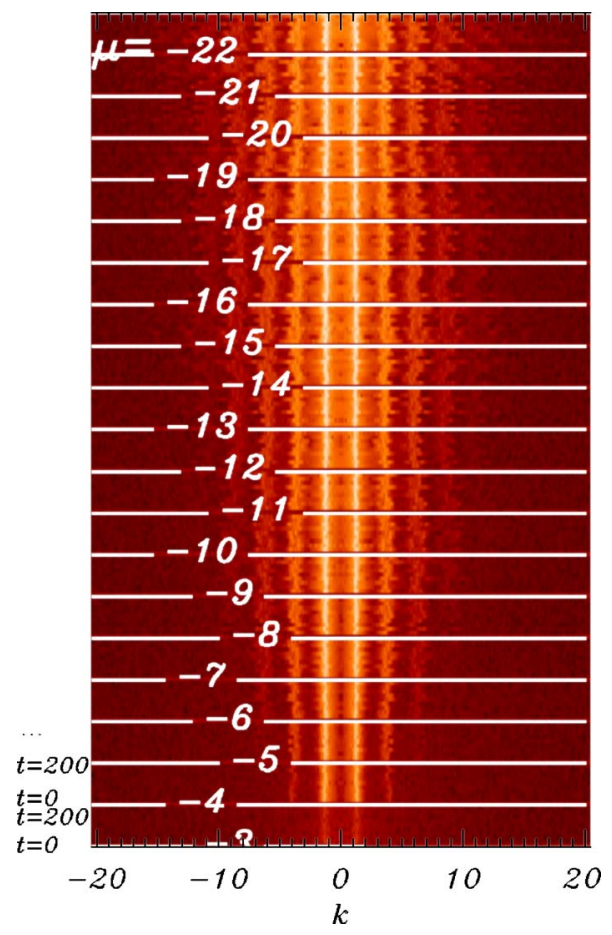

FIG. 6. (Color online) Sequence of far-field spatiotemporal diagrams decreasing the control parameter $\mu$ from -3 to -22 , shift $\Delta x=2.04$, and other parameters as in Fig. 5. A logarithmic scale is used in order to show also the spatial harmonics.

This is also in good agreement with the numerical results: an almost stationary wave is observed, for instance, in Fig. 5 for time $\gtrsim 20$ in correspondence of large wave vectors $1<k$ $<1.1$.

The wavelength spread in noise-sustained patterns is because there are different unstable modes in the system that can be linearly amplified. Through a nonlinear process, these modes are selected to form a traveling structure with finite amplitude. Figure 6 shows several spatiotemporal diagrams of the far field for decreasing values of the control parameter $\mu$ from -3 to -22 . The most evident effect increasing the distance from the threshold is the nonlinear excitation of more and more spatial harmonics within the traveling structures. The widening of the band of excited wave vectors around each harmonic can also be appreciated. For strong pump, a huge number of modes is then observed. As noted before, the NSN equation has the advantage of not developing divergences even for large values of the control parameter $(\mu=-22)$, allowing one to explore large instability regimes.

Interestingly, in Fig. 6 there are not evident signatures of the transition from convective to absolute instability that occurs for $\mu \sim-15$ for shift $\Delta x \sim 2$. As a matter of fact, noisesustained patterns are observed where stable patterns would be expected. To understand this phenomenon, we have to carefully consider the dynamics at the boundary of the system. Here we have considered a smooth super-Gaussian control parameter $\mu(x)=\mu \exp \left[-(x / \sigma)^{2 m}\right]$ with $m=10$. Then, even if within the super-Gaussian plateau the control parameter is such that the state $\phi_{0}$ is absolutely unstable, the state develops instability at the edges of the system, where the 


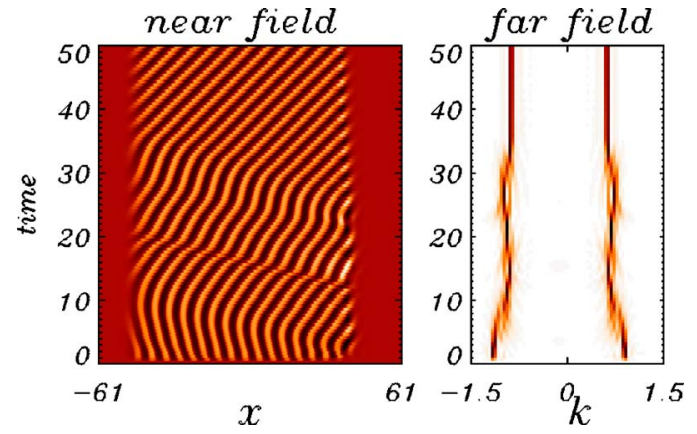

FIG. 7. (Color online) Near-field $\phi(x, t)$ and far-field $|\phi(k, t)|^{2}$ spatiotemporal plots. The control parameter has a super-Gaussian profile with value $\mu=-22$ within the plateau central region. The noise is switched off at time 25 , and the noise-sustained waves leave the system. After a longer transient, the right edge of the stable traveling pattern reaches a stationary position. Note also an interesting example of waves with phase and group velocities in the same direction $(0<t<5)$ as predicted by the linear stability analysis.

control parameter is below the absolute threshold. The noisesustained traveling waves originate, indeed, in this spatial region, where the state is convectively unstable. A noisesustained pattern is excited at the right edge of the system and travels toward the left edge, invading then the region that should be occupied by the stable pattern. Only removing any perturbation (switching off the noise source), we can observe the expected transition from convective to absolute instability. This scenario is confirmed in Fig. 7, where an initial noise-sustained pattern leaves the system when the noise is removed. In conclusion, under realistic physical conditions, as a control parameter exciting only a finite region of a system and the presence of some noise, a stable pattern cannot be observed. This can be anticipated from our linear analysis considering the convective or absolute instability in different points of the system, where the control parameter changes.

The amplitude of the noise-sustained rolls shown in Fig. 5 is plotted in Fig. 8. This amplitude increases with the distance from the instability threshold, and the square of this amplitude has approximately a linear dependence on the control parameter (inset in Fig. 8), even for very large (negative) values of $\mu$. The amplitude of the rolls has, therefore, the typical dependence $\propto \sqrt{\left|\mu-\mu_{c}\right|}$ with $\mu_{c}$ threshold of the con-

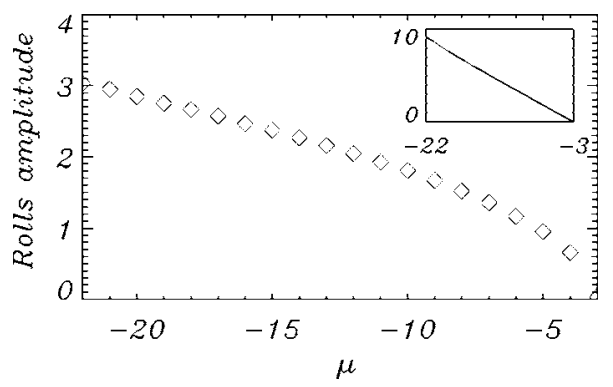

FIG. 8. Amplitude of the noise-sustained rolls of Fig. 6 for decreasing values of the control parameter. The inset shows the squared value of this amplitude. Note that the calculated threshold for the absolute instability is at $\mu_{c}=-3.37$.

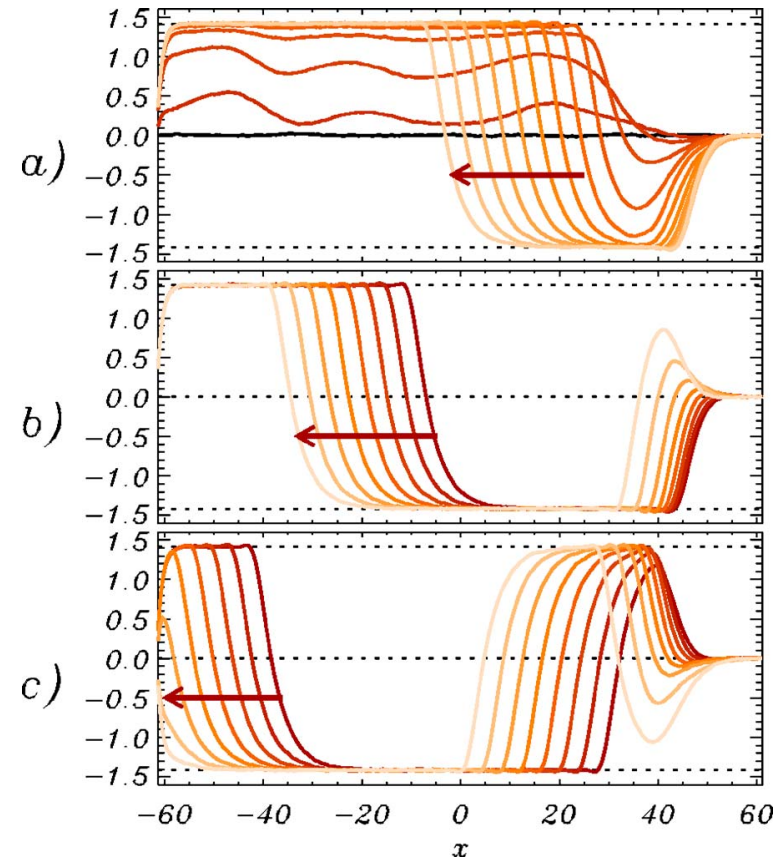

FIG. 9. (Color online) Temporal evolution of $\phi$ given by Eq. (19) in presence of an additive white Gaussian noise. The field $\phi(x)$ is plotted at different times (from dark to light colors) sampled within the intervals from $t=0$ to $t=14$ (a); from $t=15$ to $t=22$ (b); from $t=23$ to $t=30$ (c). The stationary states are plotted in dotted lines. Parameters: $\mu=3$, noise amplitude 0.01 , shift $\Delta x=2.88$. Simulation with temporal grid 0.001 , spatial grid 0.12 , and Dirichlet boundary conditions.

vective instability, characterizing this bifurcation as supercritical. Also in this case, no signatures are observed of the second transition $\left(\mu_{a}=-15\right)$, consistently with the fact that, for control parameter not vanishing only in a finite spatial region, the pattern is always sustained by noise.

\section{B. Case $\mu>1$}

The existence of different stationary solutions $\phi_{i}(i=0$, $+,-)$ for $\mu$ larger than 1 allows us to observe a very rich dynamics. In the following, we explain some aspects of the dynamics starting from our linear stability analysis. Let us consider Eq. (19) with an additive white Gaussian noise and with control parameter $\mu=3$. Then $\chi_{0}=3$ and $\chi_{ \pm}=-1 / 3$ and the stability diagram Fig. 4 tells us that the homogeneous vanishing solution is convectively unstable while the states $\phi_{+}$and $\phi_{-}$are absolutely stable. When the system evolves from a noisy initial condition, it departs from the vanishing (unstable) state toward the stable states $\phi_{ \pm}$, as expected from the linear analysis. Regions with states $\phi_{+}$and $\phi_{-}$are connected by fronts as shown in Fig. 9(a). The dynamics is characterized by a random succession of traveling fronts, Fig. 10(a), consistently with the convective instability of the vanishing state $\phi_{0}$, that is imposed by the Dirichlet boundary conditions. Indeed perturbations induced by noise grow $e x-$ ponentially from the (vanishing) right edge of the system either toward $\phi_{+}$or $\phi_{-}$. The coexistence of stable and convectively unstable states predicted by the linear stability 


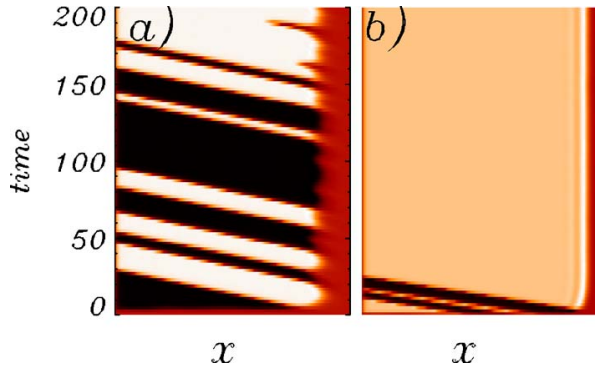

FIG. 10. (Color online) Spatiotemporal diagrams representing the evolution of $\phi$. For $\mu=3$ (a) the system exhibits noise-sustained domain walls and for $\mu=10$ (b) the system evolves toward the stable vanishing state. Other parameters as in Fig. 9.

analysis prevents the system to reach a stationary state [28]. Increasing the control parameter value from $\mu=3$, Fig. 10(a), to $\mu=10$, Fig. 10(b), the vanishing state becomes absolutely unstable. Any initial perturbation of the vanishing state arising from the noise source grows locally and after a transient dynamics, in which the fronts are advected away, the system reaches a stationary state given either by $\phi_{+}$or $\phi_{-}$. Also in this case, the linear stability analysis allows us to understand the observed deterministic evolution in comparison to the noise-sustained structure in Fig. 10(a).

Interestingly, fronts between the two stable states travel at fixed velocity, depending on the sign of the shift $\Delta x$, being $v \sim-4$ in Figs. 9(b) and 9(c). In a system with drift [Eq. (6)], this velocity would be exactly given by $v$, as these fronts would actually be stationary in the proper Galilean frame. However, the situation in presence of nonlocal coupling is far more complicated, as the nonlocality is present in any reference frame and there are several phase and group modes velocities, as discussed in Sec. II. The analysis of the motion of fronts between equally stable states is actually a question open to future investigations.

A characteristic feature of the domain walls presented above is the shape of the fronts connecting the stable solutions $\phi_{-}$and $\phi_{+}$. Because of the break of the reflection symmetry in space induced by the shift, the fronts are not symmetric (Fig. 9). As can be appreciated in more detail in Fig. 11, the leading edge of the fronts (left side in this case) is steepest and can develop spatial oscillations, while the tail of the fronts (right side, in this case) is smoother. The amplitude

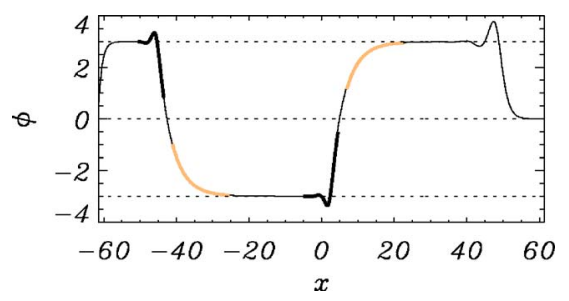

FIG. 11. (Color online) Domain walls observed in $\phi$. The asymmetry of the fronts under reflections can be appreciated comparing the leading edges (highlighted with a black thick line) and the tails (yellow thick line) of the fronts. The homogeneous solutions $\phi_{0, \pm}$ are plotted with dotted lines. The solution has been obtained from simulation of Eq. (19) (without noise), $\mu=10$, and other parameters as in Fig. 9. of the leading-edge oscillation increases with the control parameter $\mu$. The same shape of the front but for a change of sign is observed both when the front is from $\phi_{-}$to $\phi_{+}$and in the opposite case (Fig. 11).

\section{CONCLUSIONS AND OUTLOOK}

We have derived the dispersion relation for a large class of nonlinear equations with diffusion and nonlocality. These models show many different features with respect to wellknown systems in which the convective instability is caused by a drift term. A two-points nonlocality gives rise to an infinite number of oscillations in the dispersion relation, making the linear stability analysis rather complex. The convective or absolute nature of the instability of the homogeneous states can be determined by studying both local and global properties of the dispersion relation. In particular, we have shown how to find, out of an infinite number of saddle points, the subset of points necessary for the study of the instability. This subset is finite, and the saddle-point technique can be successfully used to evaluate the asymptotic behavior of perturbations. This technique also provides the stability in reference frames that are moving with respect to the laboratory without further calculations.

The second part of this paper is devoted to numerical simulations of a specific nonlocal model with a saturable nonlinearity. Critical values, convective and absolute thresholds, and phase and group velocities obtained through the linear stability analysis are in agreement with the values obtained from numerical integration. Several regimes are shown in which noise-sustained patterns or homogeneous states connected by fronts arise. The transitions between different regimes as well as the spatial structures observed in presence of several stable and unstable states need to be carefully analyzed considering boundary effects and noise effects. For instance, finite size can lead to noise-sustained states that invade the systems running over stable states. It was possible to understand this rich and sometimes surprising dynamics knowing the linear stability for any homogeneous state. A complete characterization of the observed spatial structures and of the types of bifurcations will need a nonlinear analysis, which is likely to be as challenging as the linear one. For instance, the features of drifting fronts in nonlocal diffusive systems reported for the SN equation are mainly based on numerical results. The analytical characterization of the velocity as well as shape of these fronts is an interesting subject open to investigation.

We have mentioned liquid-crystal light valves with feedback as an example of a diffusive system with two-points nonlocality [8]. It would be interesting to interpret many experimental results on these devices in view of the theoretical predictions of our analysis. Two-points nonlocality plays an important role also in optical systems with diffraction and feedback. An interesting open research line is the role of convective instability in these systems, where the analysis shown here can also be applied.

\section{ACKNOWLEDGEMENTS}

We acknowledge discussions with M. San Miguel, D. Walgraef, E. Yao D. Gomila, and E. Hernández-García. R.Z. 
is financially supported by the UK Engineering and Physical Sciences Research Council (Grant No. GR/S03898/01).

\section{APPENDIX}

Equations (14) and (15) are asymptotically satisfied at infinity by

$$
\begin{aligned}
& q_{I} \rightarrow+\infty, \quad q_{R} \sim \frac{w_{I}+\chi^{\prime} \sin q_{I}}{2 q_{I}} \\
& q_{R} \rightarrow-\infty, \quad q_{I} \sim \frac{w_{I}}{2 q_{R}} \\
& q_{R} \rightarrow+\infty, \quad q_{I} \sim\left\{\begin{array}{l}
2 n \pi-\frac{e^{-q_{R}}}{\chi^{\prime}}\left(4 n \pi q_{R}-w_{I}\right) \\
(2 n+1) \pi+\frac{e^{-q_{R}}}{\chi^{\prime}}\left[(4 n+2) \pi q_{R}-w_{I}\right]
\end{array}\right.
\end{aligned}
$$

and

$$
q_{R} \rightarrow+\infty
$$

$$
\begin{gathered}
q_{I} \sim 2 n \pi \pm\left\{\pi / 2-\frac{e^{-q_{R}}}{\chi^{\prime}}\left[-q_{R}^{2}+w_{R}-\xi^{\prime}\right.\right. \\
\left.\left.+\left(2 n \pi \pm \frac{1}{2}\right)^{2} \pi^{2}\right]\right\} \\
q_{R} \rightarrow-\infty, \quad q_{I} \sim \pm \sqrt{q_{R}^{2}-w_{R}-\xi^{\prime}}
\end{gathered}
$$

respectively. These expressions give the asymptotes for equiphase and equiamplitude paths and show that the asymptotic behavior of the equiphase paths depends on $w_{I} / \chi^{\prime}$ and $w_{I}$.
[1] J. D. Jackson, Classical Electrodynamics, 3rd ed. (Wiley, New York, 1999).

[2] M. Segev, B. Crosignani, A. Yariv, and B. Fischer, Phys. Rev. Lett. 68, 923 (1992); A. W. Snyder and D. J. Mitchel, Science 276, 1538 (1997); P. Cheben et al., Nature (London) 408, 64 (2000).

[3] L. Landau and E. M. Lifschitz, Statistical Physics (Butterworth-Heinemann, Oxford, 1996); V. M. Pérez-García, V. V. Konotop, and J. J. García-Ripoll, Phys. Rev. E 62, 4300 (2000).

[4] E. Hernández-García and C. López, Phys. Rev. E 70, 016216 (2004).

[5] Unlocking Dynamical Diversity: Optical Feedback Effects on Semiconductor Lasers, edited by D. Kane and A. Shore (Wiley, New York, 2005).

[6] M. C. Cross and P. C. Hohenberg, Rev. Mod. Phys. 65, 851 (1993).

[7] J. P. Seipenbusch T. Ackemenn, B. Schapers, B. Berge, and W. Lange, Phys. Rev. A 56, R4401 (1997); Y. Hayasaki et al., Opt. Commun. 220, 281 (2003); A. Ackemann et al., Chaos, Solitons Fractals 10(4/5), 665 (1999).

[8] P. L. Ramazza, S. Ducci, and F. T. Arecchi, Phys. Rev. Lett. 81, 4128 (1998), S. Rankin, E. Yao, and F. Papoff, Phys. Rev. A 68, 013821 (2003); L. Pastur, U. Bortolozzo, and P. L. Ramazza, Phys. Rev. E 69, 016210 (2004).

[9] E. Louvergneaux, C. Szwaj, G. Agez, P. Glorieux, and M. Taki, Phys. Rev. Lett. 93, 101801 (2004).

[10] L. D. Landau and E. M. Lifshitz, Physical Kinetics (Butterworth-Heinemann, Oxford, 1981).

[11] P. Huerre and P. A. Monkewitz, Annu. Rev. Fluid Mech. 22, 473 (1990).

[12] E. Infeld and G. Rowlands, Nonlinear Waves, Solitons and Chaos (Cambridge University Press, Cambridge, England, 1990).
[13] R. J. Deissler, J. Stat. Phys. 40, 371 (1985).

[14] R. J. Briggs, Electron-Stream Interaction with Plasmas (MIT, Cambridge, MA, 1964).

[15] J. M. Chomaz, Phys. Rev. Lett. 69, 1931 (1992); K. L. Babcock, G. Ahlers, and D. S. Cannell, ibid. 67, 3388 (1991); P. Gondret, P. Ern, L. Meignin, M. Rabaud, ibid. 82, 1442 (1999).

[16] M. Santagiustina, P. Colet, M. San Miguel, and D. Walgraef, Phys. Rev. Lett. 79, 3633 (1997); M. Santagiustina et al., Opt. Lett. 23, 1167 (1998); M. Taki, N. Ouarzazi, H. Ward, and P. Glorieux, J. Opt. Soc. Am. B 17, 997 (2000); R. Zambrini, S. M. Barnett, P. Colet, and M. San Miguel, Phys. Rev. A 65, 023813 (2002).

[17] N. Israeli, D. Kandel, M. F. Schatz, and A. Zangwill, Surf. Sci. 494, L735 (2001).

[18] O. Nekhamkina and M. Sheintuch, Phys. Rev. E 68, 036207 (2003).

[19] N. Mitarai and H. Nakanishi, Phys. Rev. Lett. 85, 1766 (2000).

[20] F. Papoff and R. Zambrini, Phys. Rev. Lett. 94, 243903 (2005).

[21] C. M. Bender and S. A. Orszag, Advanced Mathematical Methods for Scientists and Engineers (Springer-Verlag, Berlin, 1999).

[22] G. Arfken, Mathematical Methods for Physicists (Academic, New York, 1985).

[23] R. Zambrini and M. San Miguel, Phys. Rev. A 66, 023807 (2002); R. Zambrini, M. San Miguel, C. Durniak, and M. Taki, Phys. Rev. E 72, 025603(R) (2005).

[24] P. Huerre and M. Rossi, in Hydrodynamics and Nonlinear Instabilities, edited by C. Godreche and P. Manneville (Cambridge University Press, Cambridge, England, 1998).

[25] Handbock of Plasma Physics, edited by A. A. Galev and R. N. Sudan (North-Holland, Amsterdam, 1984), p. 451.

[26] L. A. Lugiato and C. Oldano, Phys. Rev. A 37, 3896 (1988); 
Van E. Wood, E. D. Evans, and R. P. Kenan, Opt. Commun. 69, 156 (1988).

[27] J. D. Murray, Mathematical Biology, 3rd ed. (Springer-Verlag, Berlin, 2002).

[28] We note that, even if the spatial structures shown in Figs. 9-11 have been obtained assuming Dirichlet boundary conditions and a uniform control parameter; a similar scenario appears also when considering a super-Gaussian $\mu(x)$, even if for slightly different reasons. 